\title{
A MODEL OF TOUGHENING EFFECTS IN WHISKER-REINFORCED COMPOSITES
}

\author{
R. G. Hoagland ${ }^{1}$ and C. H. Henager, jr. ${ }^{2}$ \\ 1 Department of Mechanical and Materials Engineering \\ Washington Sta:e University \\ Pullman, Washington 99164-2920 \\ 2Pacific Northwest Laboratory \\ Richland, Washington 99352
}

\begin{abstract}
A numerical approach is presented that lends itself to modeling the screening or antiscreening effects due solely to modulus differences of a discrete array of whiskers in an elastic matrix. The method is applied to single whiskers, and to examining the issue of whisker orientation on the toughness of ceramic composites. The model results indicate that crack-tip shielding due to modulus defect interactions occurs when the reinforcement has a higher modulus than thic matrix material, and that anti-shielding occurs for the opposite case. Results for a single whisker located at the crack-tip (maximum effect) indicate that the crack-tip stress intensity is reduced by about $10 \%$ when a modulus ratio of four is assumed. Calculations performed with whisker arrays demonstrate pronounced effects of whisker orientation on the crack-tip screening, being larger for whiskers oriented perpendicular to the crack plane, as expecteri. Ordered whisker arrays produce larger and more uniform screening than do random whisker arrays.
\end{abstract}

\section{Introduction}

Most single phase ceramics that have useful high temperature properties suffer from a lack of low temperature ductility and toughness. Multiphase microstructures can have better toughness depending on the relative properties of the phases, their geometric arrangement and the strength of their interfaces. Among the many possibilities, the addition of strong, high modulus fikers or whiskers to brittle materials has, in many cases, yielded attractive improvements in toughness.

Several types of toughening mechanisms in such fiber-reinforced materials have been advanced to explain the improvements. One example is crack bridging[1-5] that occurs when a crack in the brittle matrix grows beyond one or more whiskers that are oriented 
more or less perpendicular to the crack plane. Mechanically, the system can be regarded as a kind of Dugdale zone, in which intact whiskers are replaced by a corresponding set of pinching forces[4] acting to close the crack and, therefore, diminish the crack tip stress intensity. This toughening effect is analogous to that created by ligament formation during fracture of metals[6]. If the whisker/matrix interface is strong, the bridging whiskers become highly stressed where they span the crack opening. Sliding along the whisker/matrix interface helps smooth the stress distribution somewhat and absorbs energy. This allows the whiskers to survive larger crack openings and to exert larger toughening forces. Another mechanism that has been suggested is crack deflection[7,8] in which the crack path becomes tortuous as the crack moves around whiskers and along the interfaces.

As short fibers, whiskers can also influence toughness if they have elastic properties different from the matrix, in which case they become sources of internal stress (the socalled modulus defect) when an external stress is applied. Sources of internal stress, as, for example, dislocations, microcracks, and dilatant centers, will, in general, affect the toughness of a material via the influence they exert on the crack tip driving force [9-12]. If, in the presence of a crack, the defect (source of internal stress) acts to decrease $\mathrm{K}$, the effect is referred to as screening or shielding, and the apparent toughness is increased because the applied $\mathrm{K}$ (or equivalently the applied loading) has to be increased to obtain crack extension $[9,10]$. Loss of apparent toughness is caused by antiscreening. The potential contribution to the toughness from whiskers as modulus defects has apparently not been examined in detail.

In the present paper, we present a a numerical procedure that approximately models the influence of one or more whiskers on the crack tip field for an otherwise elastic solid. The method we use is similar to that employed by Hoagland and Embury[13] who used it to examine the effects of microcracking and by Bowling, et al. [14] in a study of toughening contributions from both microcracks and dilatant centers. Because we retain the discrete character of the defects, this procedure may appear to contrast with continuum approaches like that of Budiansky, et al. [15] but, in fact, the latter is a bound of the former in the case when the scale permits the smearing of the defects into a continuous distribution of infinitesimal defects. On the other hand, a discretized picture incorporates a measure of the point-to-point variability in the material and the consequences of different sequences of discrete events. 


\section{Computational Method}

A great deal of computational convenience is gained by first considering the model as twodimensional as shown in Figure 1.The crack length and other in plane dimensions are regarded as much larger than the whisker size and spacing. The 2-D assumption is reasonable if, in the thickness direction, the slab is also much larger than the whisker size and spacing, and the whiskers in this 2-D model can then be regarded as through-thethickness averages of a 3-D population.

We have also approximated the 2-D shear lag stresses for an elastically dissimilar whisker in an infinite medium to further simplify the problem. Figure 2 compares schematically three distributions, a shear lag theory prediction [16], a stick-slip distribution in which sliding occurs at a constant shear stress [16], and the linear approximation to the shear stresses at the whisker/matrix interface used in this study. The shear lag theory has a shear stress that varies along the length of the whisker as $\sinh (\mathrm{nx} / \mathrm{r})$ where $\mathrm{x}$ is the distance along the whisker axis with the origin at the midlength position. To obtain the stresses surrounding a whisker subject to a linear shear stress distribution in an otherwise infinite homogeneous elastic medium, we consider a Green's function for a segment of the force acting parallel to and along the whisker axis as represented in Figure 3. If position along a whisker relative to its midpoint is $t$ and the strength of the force (per unit length) acting along the whisker is $S$, the stresses in the surroundings due to the forces acting on segment dt at $t$ are

$$
\begin{aligned}
& d \sigma_{x x}=\frac{S d t}{2 \pi(k+1)}\left\{(k-1) \frac{y-t}{t^{2}+2 y t+r^{2}}-\frac{4 x^{2}(y-t)}{\left(t^{2}+2 y t+r^{2}\right)^{2}}\right\} \\
& d \sigma_{y y}=\frac{S d t}{2 \pi(k+1)}\left\{-(k+3) \frac{y-t}{t^{2}+2 y t+r^{2}}-\frac{4 x^{2}(y-t)}{\left(t^{2}+2 y t+r^{2}\right)^{2}}\right\} \\
& d \sigma_{x y}=\frac{S d t}{2 \pi(k+1)}\left\{(k+3) \frac{x}{t^{2}+2 y t+r^{2}}-\frac{4 x^{3}}{\left(t^{2}+2 y t+r^{2}\right)^{2}}\right\}
\end{aligned}
$$


where $k=3-4 \nu$ and $r^{2}=x^{2}+y^{2}$ and $x$ and $y$ are as defined in Figure 3. The distribution is made linear by setting $S=\psi t$, where $\psi$ is defined below. Integrating over the length of the whisker from $-\ell$ to $+\ell$ gives the net stresses at a $x, y$-field point in the infinite $2 D$ space due to a whisker of length $2 \ell$ at the origin. Some brevity is provided by using nondimensional coordinates, i.e., by replacing $x$ by $x \ell$ and $y$ by $y \ell$, giving for the stresses

$$
\begin{aligned}
& \sigma_{x x}=\frac{\psi}{2 \pi(k+1)}\left\{(k-1)\left[-y \ln \frac{R_{1}}{R_{2}}+x\left(\theta_{1}-\theta_{2}\right)-2 \mid\right]-2 x^{2}\left[\left(\frac{1}{R_{1}^{2}}+\frac{1}{R_{2}^{2}}\right)-\frac{\left(\theta_{1}-\theta_{2}\right)}{x}\right]\right\} \\
& \sigma_{y y}=\frac{\psi}{2 \pi(k+1)}\left\{-(k+3)\left[-y \ln \frac{R_{1}}{R_{2}} \therefore x\left(\theta_{1}-\theta_{2}\right)-2 \mid\right]+2 x^{2}\left[\left(\frac{1}{R_{1}^{2}}+\frac{1}{R_{2}^{2}}\right)-\frac{\left(\theta_{1}-\theta_{2}\right)}{x}\right]\right\} \\
& \sigma_{x y}=\frac{\psi x}{2 \pi(k+1)}\left\{-(k+3)\left[\ln \frac{R_{1}}{R_{2}}+\frac{y\left(\theta_{1}-\theta_{2}\right)}{x}\right]+2\left[\frac{r^{2}+y}{R_{2}^{2}}-\frac{r^{2}-y}{R_{1}^{2}}+\frac{y\left(\theta_{1}-\theta_{2}\right)}{x}\right]\right\}
\end{aligned}
$$

where $R_{1}^{2}=1-2 y+r^{2}, R_{2}^{2}=1+2 y+r^{2}, \theta_{1}=\tan ^{-1}((1-y) / x), \theta_{2}=-\tan ^{-1}((1+y) / x), \ell$ is half the whisker length, and it is to be understood that $x, y, r$ and $R_{i}$ are nondimensional.

The magnitude of the stresses in the matrix surrounding the whisker is controlled by the coefficient $\psi$ which, in turn, must be determined by the response of the system (whisker and matrix) to an applied stress parallel to the whisker axis. In what follows we have neglected interaction with shear components. The magnitude of this response can be determined in the following way. Consider the force supported by the whisker

$$
F=\ell \int_{-\alpha / 2}^{q / 2} \sigma_{y y} d x=\psi l a \frac{(k+3)}{(k+1)}
$$

where $\mathrm{a}$ is the whisker diameter. Using the condition that a uniform normal stress applied at $\pm y=\infty$ in the matrix, $\sigma_{m}$, produces a stress in the whisker of $\sigma_{\mathrm{f}}$ and that the strain in the matrix approximately matches that of the whisker, we can set $F / \ell_{a}=\sigma_{f}-\sigma_{m}$ and gives for $\psi$ 


$$
\psi=\sigma_{m}\left(\frac{E_{1}}{E_{m}}-1\right) \frac{(k+1)}{(k+3)}
$$

where $E_{f}$ and $E_{m}$ are the Young's modulus of the whisker and matrix, respectively. Thus, the strength of the stresses generated by the whisker are linearly related to the nominal stress applied to it, the modulus ratio, and inversely to the whisker length.

Equation 2 provides the means for accommodating the presence of an arbitrarily oriented whisker by superposing these stresses everywhere, at least at those locations of interest such as other whiskers and along the crack faces, after transforming the stresses to account for the rotated coordinates. The conditions involved in the derivation of equations 2 and 4 , strictly confine equation 2 to a $2 \mathrm{D}$ whisker immersed in a uniform stress. A further simplification we have adopted is to neglect variations in applied stress over the length of the whisker, and set the component of the normal stress acting parallel to the whisker axis at the whisker mid point equal to $\sigma_{\mathrm{m}}$. In view of the other approximations, this assumption probably has little substantive influence on the results except for whiskers which may be subject to stresses that vary drastically over their length. Therefore, this analysis is most appropriate to short whiskers.

The situation in which more than one whisker exists and a crack is also present is treated numerically in the manner described in more detail in references 13 and 14 . Briefly, each whisker experiences the crack tip stress field, the magnitude of which is determined by the two stress intensities, $\mathrm{K}_{1}$ and $\mathrm{K}_{2}$, and the stress fields of all other whiskers in the model. The traction free surfaces which define the crack are maintained by applying point forces along closely spaced points on the crack faces as needed to remove the tractions created by the sum of the stresses, from equation 2 , of all the whiskers. These point forces change the nominal stress which each whisker experiences, and, in addition, change the stress intensities. If, in the crack coordinates, $\sigma_{y y}$ and $\sigma_{x y}$ are the tractions produced by the whiskers, and $\delta c$ is an increment of length along the crack face located at the ith point a distance $c_{i}$ from the crack tip, the change in mode 1 and 2 stress intensity factors are 


$$
\Delta K_{1}=2 \sum \frac{\sigma_{y y} \delta c}{\sqrt{2 \pi c_{i}}}
$$

$$
\Delta K_{2}=2 \sum \frac{\sigma_{x c} \delta c}{\sqrt{2 \pi c_{i}}}
$$

where the sums are carried out to a distance sufficiently far from the tip that the tractions have decayed to reasonably small levels. In fact the range of the stress field of a whisker is rather short, being on the order of the whisker length, and therefore for the models discussed here which contained up to a few hundred whiskers in a region up to 20 whisker lengths in extent, we found that the tractions became quite small at distances of greater than about 10 whisker distances from the crack tip.

The calculation iteratively cycles over all points, whiskers and crack faces, using the stress increment at each point in a cycle as the Green's function for the next iteration cycle. When the largest change in the stresses at any point becomes less than a preset value, the calculation is considered to have converged to a solution that identifies the total stress acting on each whisker and produces a reasonably stress free crack. Typically, we find that the convergence is rapid, normally about three iterations to produce changes which are less than $0.1 \%$ of the stress at a point.

In the following section, we present results that examine some of the effects produced by one whisker, as well as the consequence of moving a crack through an array of whiskers whose position and orientation were determined by a random number generator. Because of the approximations discussed above, these results should be regarded as qualitative and as representing trends.

\section{Results}

\section{Single Whisker}

We present first some results demonstrating the influence of a single whisker on the crack tip stress field. The magnitude of that interaction is a function of three coordinates, $r, \theta$, and $\phi$, shown in Figure 1, where the first two define the position of the midpoint of the 
whisker in crack tip coordinates, and the last, the orientation of the whisker axis relative to the $x$-axis, the crack propagation direction. In Figures 4 - 6 are shown the computed dependence of the changes in the two stress intensities, $\Delta \mathrm{K}_{1}$ and $\Delta \mathrm{K}_{2}$, on $\phi$. In these results, since the magnitude of the effects scale with the applied $K$, the dependence is expressed in terms of the relative change, $\Delta \mathrm{K}_{\mathfrak{l}} /\left(\psi_{0} \mathrm{~K}_{1}\right)$, where $\mathrm{K}_{1}$ is the applied stress intensity and $\psi_{0}$ is the group of elastic constants multiplying $\sigma_{\mathrm{m}}$. The applied mode 2 stress intensity was zero. In these figures, the whisker was located at a radial distance of 2 and at several values of $\theta$.

These results (Figures 4 - 6) indicate that, as $\theta$ increases from 0 , the mode 1 contribution evolves from screening for all whisker orientations, to a more complex behavior involving some orientations that cause screening and others that are antiscreening at $\theta=\pi / 2$. For a whisker with a modulus ratio of 5 , the maximum screening occurs ahead of the crack tip corresponding to a $10 \%$ decrease in $\mathrm{K}_{1}$. Also, the mode 2 contribution at most positions is of the same order as the mode 1.

One way to gain an appreciation for the spatial dependence of screening is to assume that the number of whiskers is very large and that their orientations are uniformly distributed. An effective, or average, screening contritution can be obtained which removes the $\phi$ dependence by integrating over $\phi$ from $0 t, \pi$ and dividing by $\pi$. The resulting average value of the relative change in the mode $1 \mathrm{~K}$ is given by

$$
\frac{\overline{\Delta K}}{K}=\frac{1}{\pi K} \int_{0}^{\pi} \Delta K d \phi
$$

The $\theta$-dependence of the average computed by numerical integration for the $r=2$ results is shown in Figure 7. In terms of mode 1, Figure 7 shows that whiskers tend to screen for $0<\theta<93$ and antiscreen at $93<\theta<153$. However, there is a net screening effect $\left(\triangle K_{1} / K_{1} 0\right.$ $<0$ ) at this distance from the crack tip, for all values of $\theta$. Also, while we display the results for $\theta>153$ in Fig. 7, the assumption of matching strains in the matrix and whisker does not apply in this range because of the discontinuity in displacements across the crack faces and the fact that, for some $\phi$, the whiskers straddle the crack. In these cases, the pinching forces mentioned above would provide a better description of the effects of the whiskers. 
Figure 8 displays examples of the radial dependence of the average mode 1 contribution for several values of $\theta$. The rather rapid decrease in the effect of the whisker with increasing distance form the crack tip is evident in this figure, as is the very weak interaction that occurs with the crack for whiskers located directly above or below the crack. The strongest interaction occurs for whiskers ahead of the crack where the direction of the effect is screening.

\section{Whisker Arrays}

By monitoring the mode 1 and 2 stress intensities as the crack tip is extended in small increments through an array of whiskers, the consequences of various multiple-whisker arrangements on toughness can be explored. Such calculations are conducted iteratively as described above. In the results that follow, the aspect ratio (2\&/a) was chosen to be 100 and none of the whiskers were allowed to cross the crack plane. Therefore, the results show effects solely due to modulus defect interactions. Also, all of the whiskers have the same $\psi_{0}(>0)$. Whiskers for which $-1<\psi_{0}<0$, would produce similar results except that the changes in the stress intensity would have opposite signs; i.e., screening would become antiscreening and vice versa.

Figure 9 shows results for a crack extending from $x=-6$ to $x=+6$ through a randomly positioned array of whiskers. There are 100 whiskers involved in this array, which, for an aspect ratio of 100 and the size of the model used, corresponds to a rather modest volume fraction of $4 \%$. Three sets of calculations are presented here. In one case, the net change in mode 1 stress intensity for randomly oriented whiskers (produced from a uniformly distributed set of random numbers) is shown and for most, but not all, of the segment of extension, the effect is screening, i.e. $\Delta \mathrm{K}_{1}<0$. The other two sets of results are for nonrandom arrays in which the whiskers have a preferred orientation produced by a normally distributed set of random numbers with a standard deviation of $\pi / 10$ and a mean of $\pi / 2$ in one case (perpendicular orientation) and zero (parallel orientation) in the other. For this particular whisker array there is a distinct advantage of a perpendicular orientation. In fact, over a significant portion of the crack extension distance in the parallel orientation, there exists antiscreening, although a part of this antiscreening is an artifact of the model, as will be described below. A similar set of results for another randomly positioned array containing 200 whiskers but with the same volume fraction is shown in Figure 10. 
When the whisker array is spatially non-random there emerges a stronger indication of the advantage of whisker alignment, as shown in Figure 11. Here we show the results of a crack which has been extended through a square array of perfectly oriented whiskers, oriented perpendicular to the crack plane in one case and parallel in the other. Screening by the perpendicular array is considerably geater. A stress intensity which oscillates with the period of the array spacing is also evident.

A distinct trend of increasing $\mathrm{K}$ with crack length is also apparent in Figure 11. This trend derives from the fact that, in this and the previous models, the whiskers are contained in a finite size region and that the crack tip field, therefore, senses the lack of whiskers beyond the boundaries of the model. In other words, for a short crack length there are a preponderance of whiskers which lie in the strong screening zone ahead of the crack while as the crack advances more whiskers enter the zone of mixed interaction. This effect also demonstrates that, while the range of the effect exerted by a whisker decreases fairly quickly with distance from the crack tip, there are consequences of ignoring the tiny contributions from an infinite region. Indeed, if the size of the model were increased without limit this trend would disappear. While we have not done so here, we could correct these results by including an additional contribution to the local $\mathrm{K}$ that would remove this trend by treating the material outside the model as containing a continuum of infinitesimal whiskers and integrating over that space. This correction is discussed in more detail by Bowling et al. [14].

\section{Discussion}

A numerical approach has been presented that lends itself to modeling the screening or antiscreening effects due solely to modulus differences of a discrete array of whiskers in an elastic matrix. We have applied the method to single whiskers, and to examining the issue of whisker orientation on toughness. The model results indicate that crack-tip shielding due solely to modulus defect interactions occurs when the reinforcement has a higher modulus than the matrix material, and that anti-shielding occurs for the opposite case. Results for a single whisker located at the crack-tip (maximum effect) indicate that the crack-tip stress intensity is reduced by about $10 \%$ when a modulus ratio of four is assumed. Calculations performed with whisker arrays demonstrate pronourced effects of whisker orientation on the crack-tip screening, being larger for whiskers orisnted perpendicular to the crack plane, as expected. Ordered whisker arrays produce larger and more uniform screening than do random whisker arrays. 
There are many additional aspects of the problem which we have not addressed including crack bridging, interface slip/failure, whisker fracture and others. However, the effects which these additional aspects of the problem would exert could be accommodated relatively easily. For example, a critical stress criterion could be invokeri that would either limit the strength of the whisker, i.e., its $\psi 0$, or to remove it, e.g., to rnimic complete failure of the interface, or to change its length simulating whisker fracture. Thus, this kind of approach creates the opportunity for exploring a wide range of problems and microstructures.

To compare the toughening contributions of whiskers treated as modulus defects with other ways they may affect toughness, such as by bridging the crack, requires some consideration of the several factors of size, aspect ratio, and modulus ratio, as well as distribution. For modulus ratios of four, an aspect ratio of 100 , and a volume fraction of $4 \%$, these results suggest that toughness increases of about $10 \%$ above that of the matrix should result. This is perhaps not as strong a screening effect as would derive from an equivalent volume fraction of bridging whiskers, but it is important to recognize that many whiskers that may bridge a crack are not oriented perpendicular to the crack plane and therefore will be subject to large bending moments in addition to tension created that resists the crack opening displacements. Such unfavorably aligned whiskers would not be able to play a significant bridging role.

\title{
Acknowledgments
}

The authors would like to acknowledge the useful discussions and encouragement by Dr. R. H. Jones and the discussions with 'rrof. J. P. Hirth. One of us, R. G. H., would also like to gratefully acknowledge the support of the Pacific Northwest Laboratory in this work. This work was supported by the Office of Basic Energy Sciences, Division of Materials Sciences, U.S. Department of Energy under Contract DE-AC06-76RLO 1830.

\section{DISCLAIMER}

\begin{abstract}
This report was prepared as an account of work sponsored by an agency of the United States Government. Neither the United States Government nor any agency thereof, nor any of their employees, makes any warranty, express or implied, or assumes any legal liability or responsibility for the accuracy, completeness, or usefulness of any information, apparatus, product, or process disclosed, or represents that its use would not infringe privately owned rights. Reference herein to any specific commercial product, process, or service by trade name, trademark, manufacturer, or otherwise does not necessarily constitute or imply its endorsement, recommendation, or favoring by the United States Government or any agency thereof. The views and opinions of authors expressed herein do not necessarily state or reflect those of the Utited States Government or any agency thereof.
\end{abstract}




\section{References}

1. B. Budiansky, J. W. Hutchinson, and A. G. Evans, "Matrix Fracture in FiberReinforced Ceramics", J. Mech. Phys. Solids, 34, 167-189 (1986).

2. M. Ruhle, B. J. Dalgleish, and A. G. Evans, "On the Toughening of Ceramics by Whiskers", Scripta Met., 21, 681-686 (1987).

3. D. B. Marshall and A. G. Evans, "The Tensile Strength of Uniaxially Reinforced Ceramic Fiber Composites", Fracture of Ceramics, Vol. 7, ed. by R. C. Bradt, et al., Plenum, N.Y., 1-15 (1986).

4. A. G. Evans and R. M. McMeeking, "On the Toughening of Ceramics by Strong Reinforcements", Acta Metall., 34, 2435-2441 (1986).

5. D. B. Marshall, B. N. Cox, and A. G. Evans, "The Mechanics of Matrix Cracking in Brittle-Matrix Fiber Composites", Acta Metall., 33, 2013-2021 (1985).

6. R. G. Hoagland, A. R. Rosenfield, and G. T. Hahn, "Mechanisms of Fast Fracture and Arrest in Steels", Met. Trans, 3, 123-136 (1972).

7. K. T. Faber and A. G. Evans, "Crack Deflection Processes-I. Theory", Acta Metall., $31,565-576$ (1983).

8. P. F. Becher, T. N. Tiegs, J. C. Ogle, and W. H. Warwick, "Toughening of Ceramics by Whisker Reinforcement", Fracture of Ceramics, Vol. 7, ed. by R. C. Bradt, et al., Plenum, N.Y., 61-73 (1986).

9. R. Thomson and J. E. Sinclair, "Mechanics of Cracks Screened by Dislocations", Acta Metall., 30, 1325 (1982).

10. R. Thomson, "Brittle Fracture in a Ductile Material with Application to Hydrogen Embrittlement", J. Mater. Sci., 13, 128-142 (1978).

11. J. P. Hirth, R. G. Hoagland, and C. H. Popelar, "On Virtual Thermodynamic Forces on Defects and Cracks", Acta Metall., 32, 371-379 (1984).

12. R. G. Hoagland and J. P. Hirth, "Dilatant Center-Crack Tip Interaction Effects", J. Am. Cer. Soc., 69, 530-533 (1986).

13. R. G. Hoagland and J. D. Embury, "A Treatment of Inelastic Deformation Around a Crack Tip due to Microcracking", J. Am. Cer. Soc, 63, 404-410 (1980).

14. G. D. Bowling, K. T. Faber, and R. G. Hoagland, "Computer Simulations of RCurve Behavior in Microcracking Materials", J. Am. Cer. Soc., 70, 849-854 (1987).

15. B. Budiansky, J. W. Hutchinson, and J. C. Lambropoulos, "Continuum Theory of D latant Transformation Toughening in Ceramics", Int. J. Solids Struct., 19, 337-355 (1983).

16. M. R. Piggott, Load Bearing Fibre Composites, Pergamon, N.Y., 83-99 (1980). 


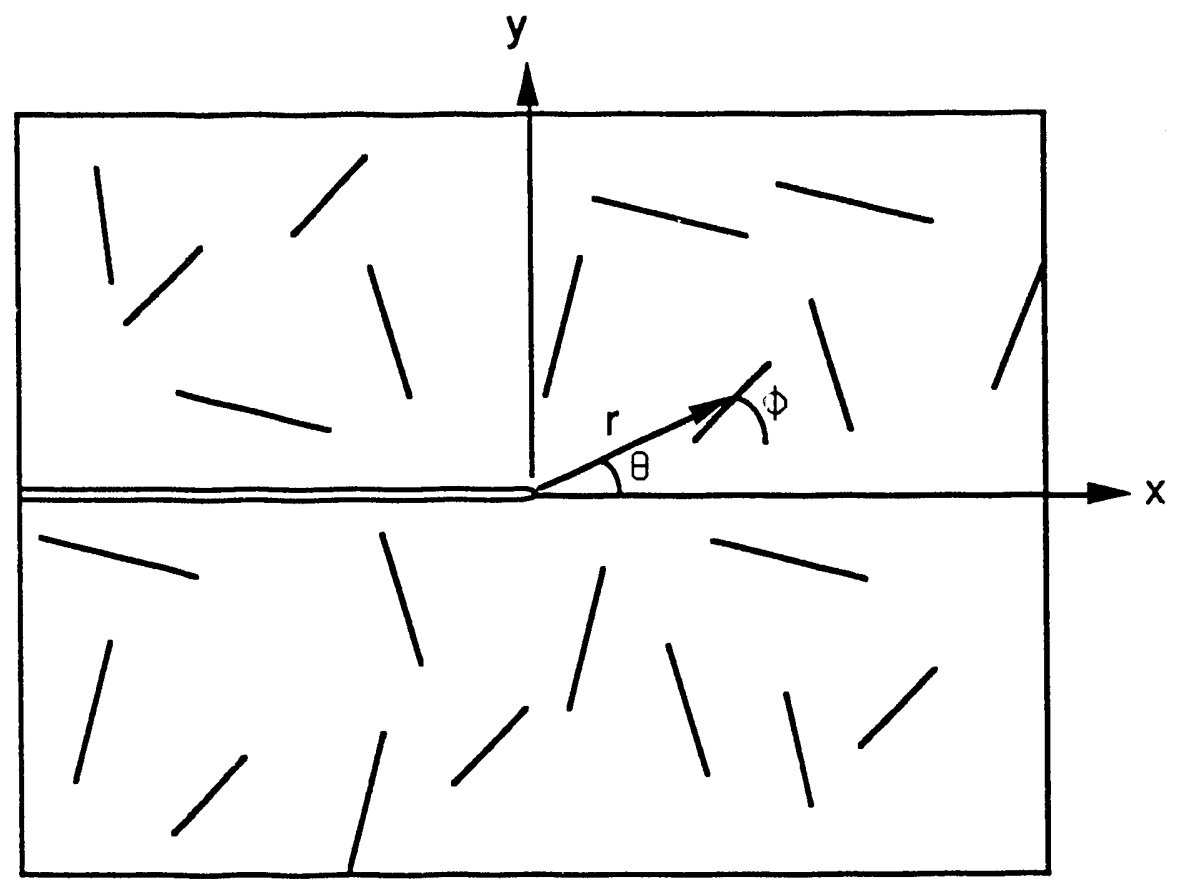

Figure 1. Schematic of the model configuration. 


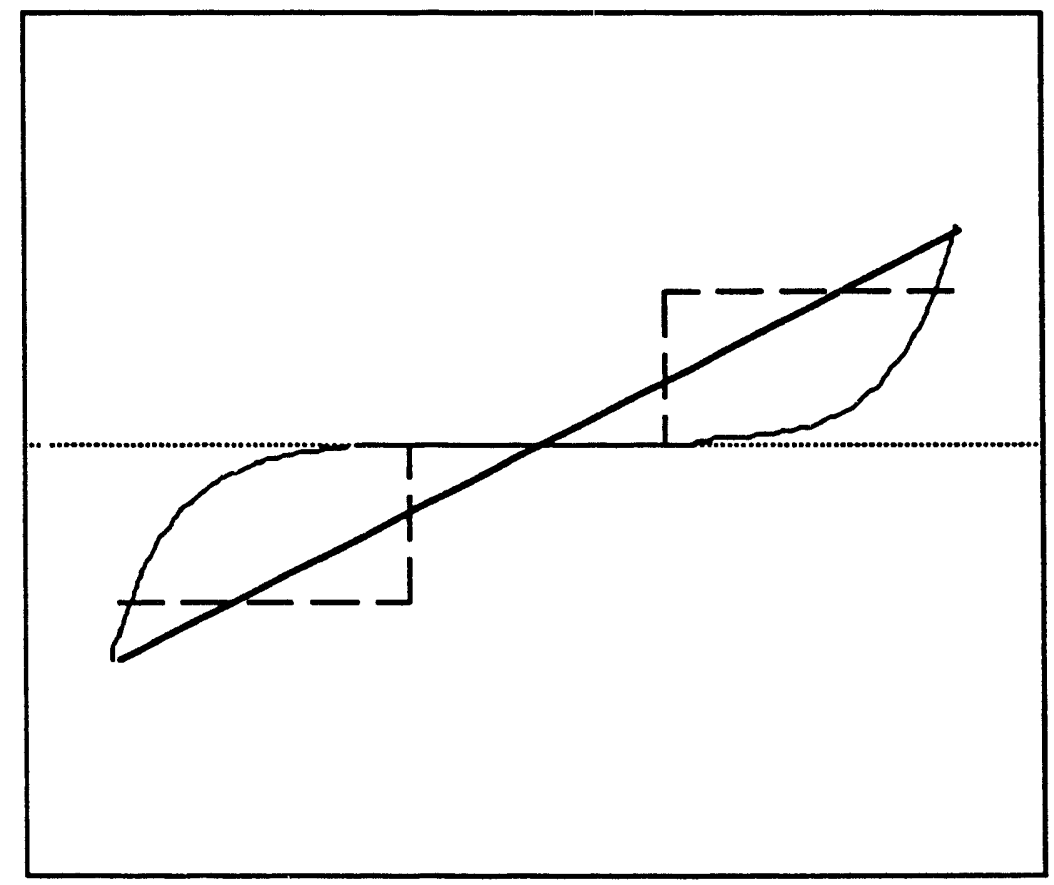

Figure 2. Schematic shear stresses at whisker/matrix interface from shear lag theory (curve) and the assumed linear stress distribution used in model described here. The dash line represents another alternative distribution in which sliding occurs at the interface at a constant shear stress [16].

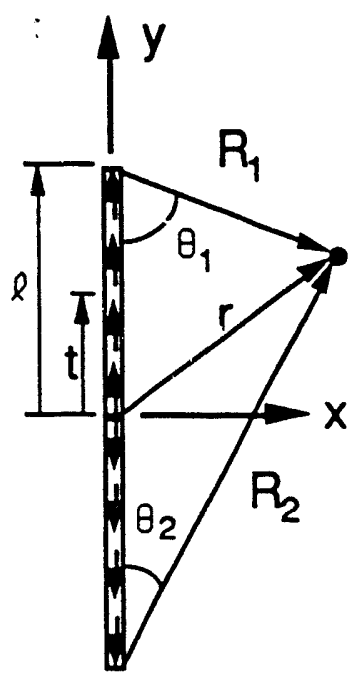

Figure 3. The action of the whisker on the matrix is represented by a distribution of forces of strength $S$ so that the segment dt contains force of magnitude Sdt. The other symbols are defined in the text. 


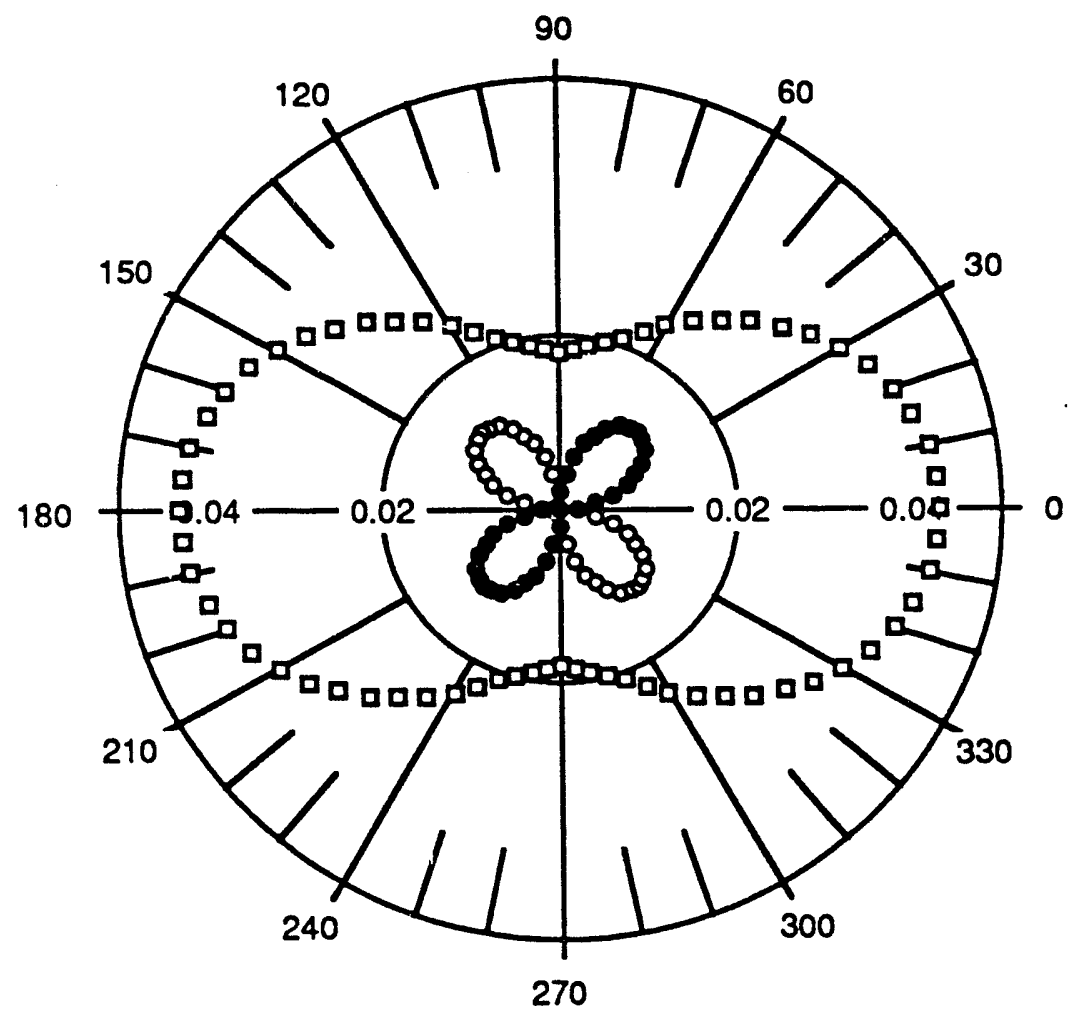

Figure 4. A polar plot showing the relative change in the stress intensities, $\Delta \mathrm{K}_{1} /\left(\psi_{0} \mathrm{~K}_{1}\right)$ (square symbols) and $\Delta \mathrm{K}_{2} /\left(\psi_{0} \mathrm{~K}_{1}\right)$ (circular symbols) as a function of the whisker axis orientation, $\phi$. Negative and positive values are represented by open and closed symbols, respectively. The whisker midpoint is at located at $r=2$, and $\theta=0$. 


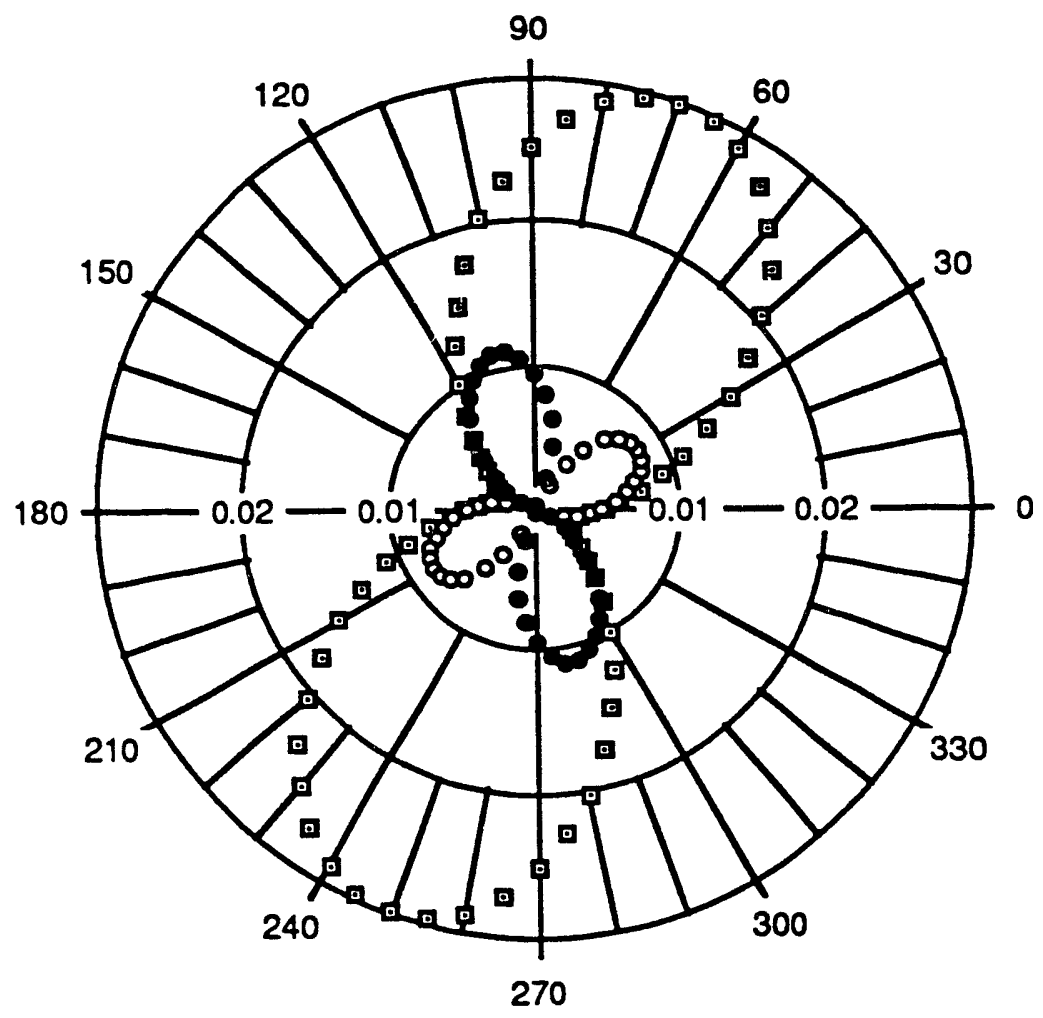

Figure 5. Same as Figure 4 except $\theta=\pi / 4$.

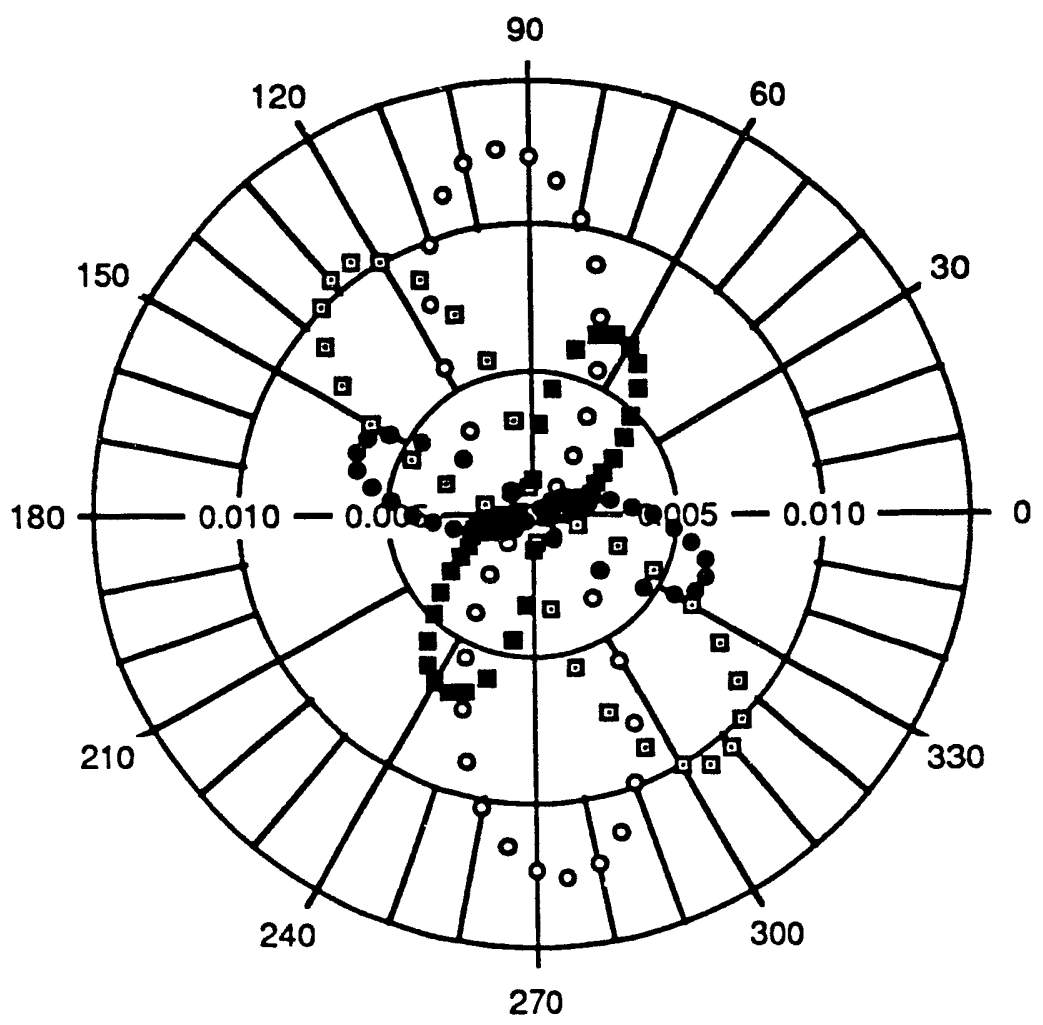

Figure 6. Same as Figure 4 except $\theta=\pi / 2$. 


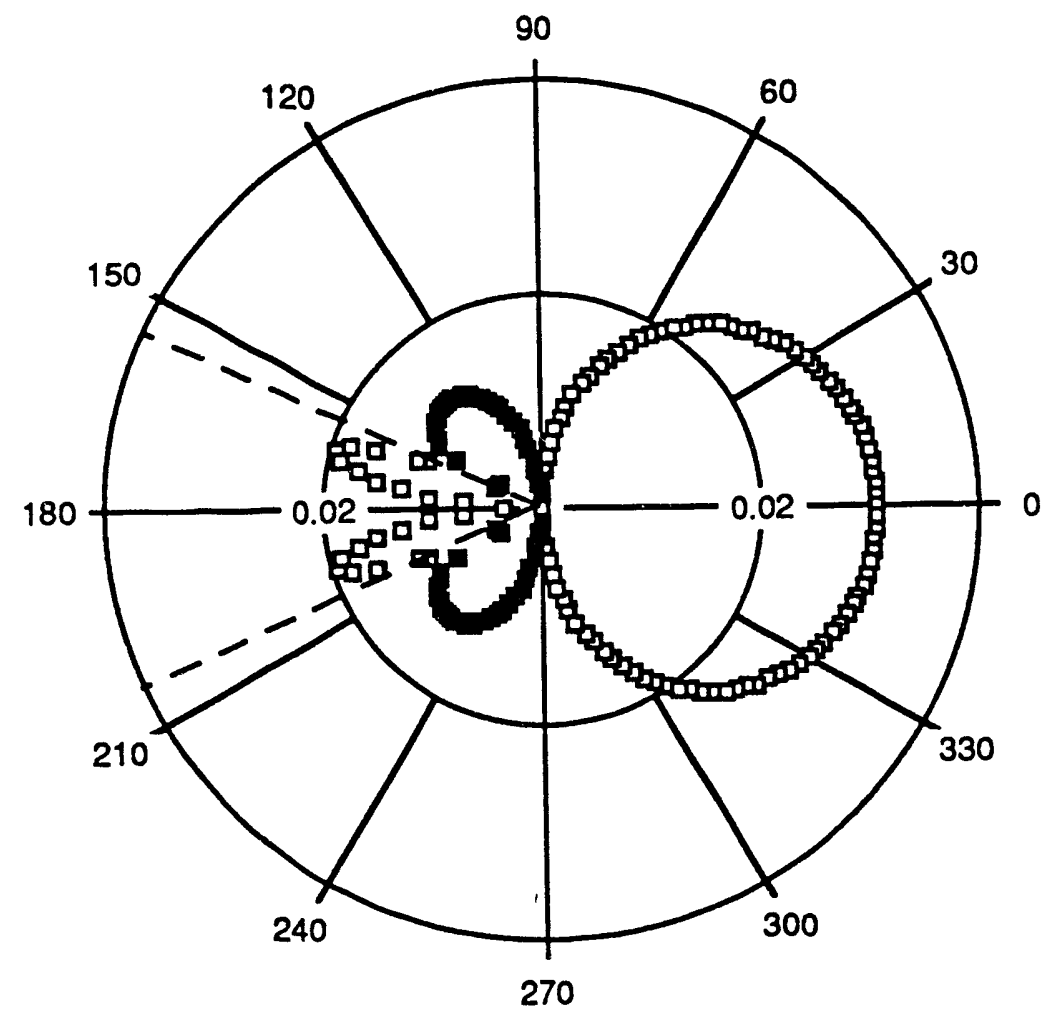

Figure 7. A polar plot of the average mode 1 contribution, $\Delta \mathrm{K}_{1} / \mathrm{K}_{1}$, as a function of the position angle, $\theta$, for $r=2$. Again, open points are negative and closed points, positive. Results in the range $153<\theta<206$ (between the dashed lines) do not reflect the fact that the whisker intersects the crack for some $\phi$ in this range. 


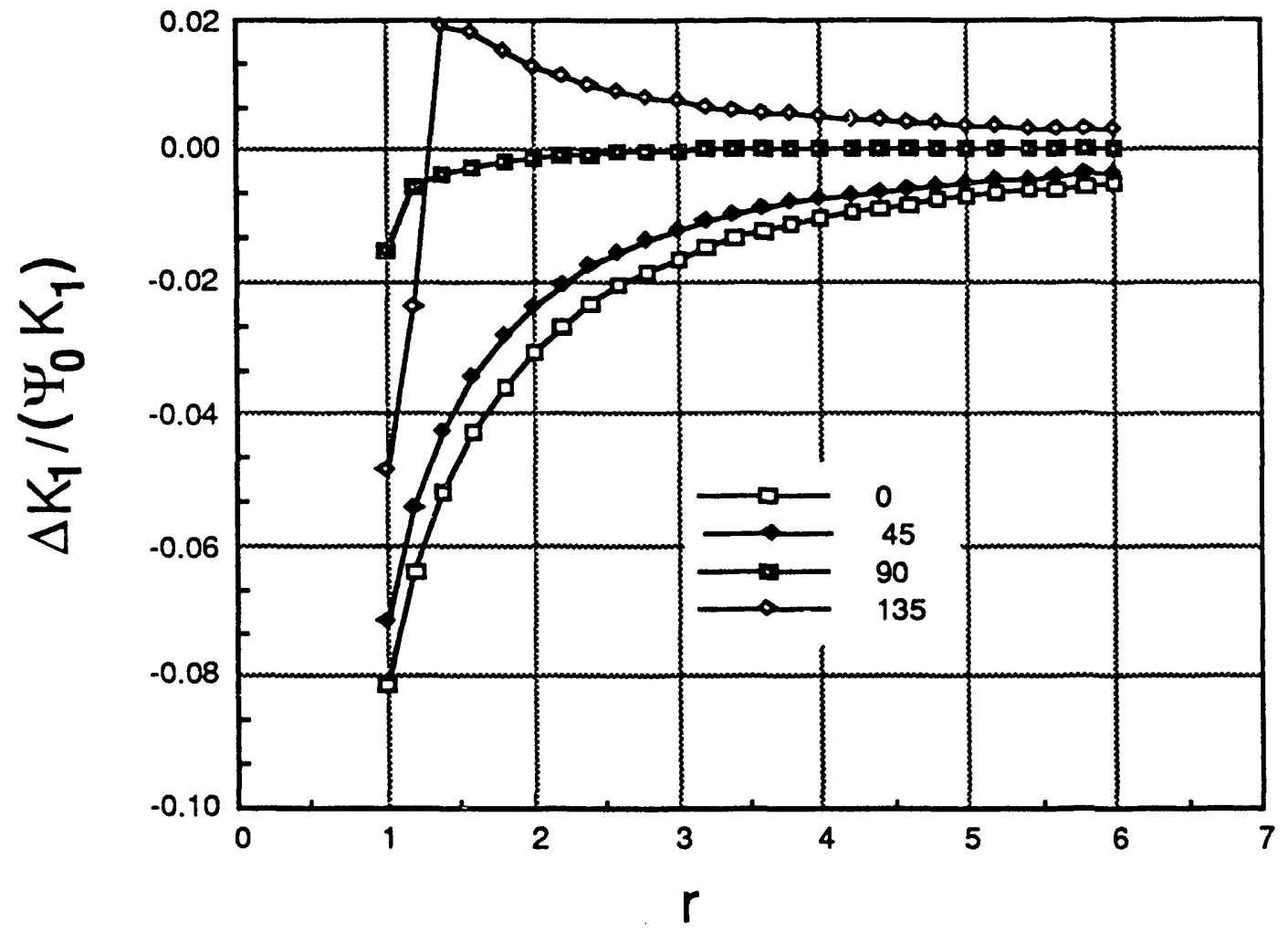

Figure 8. Radial dependence of the average mode 1 contribution, $\Delta \mathrm{K}_{1} / \mathrm{K}_{1}$, for several values of the position angle, $\theta$, for a single whisker. 


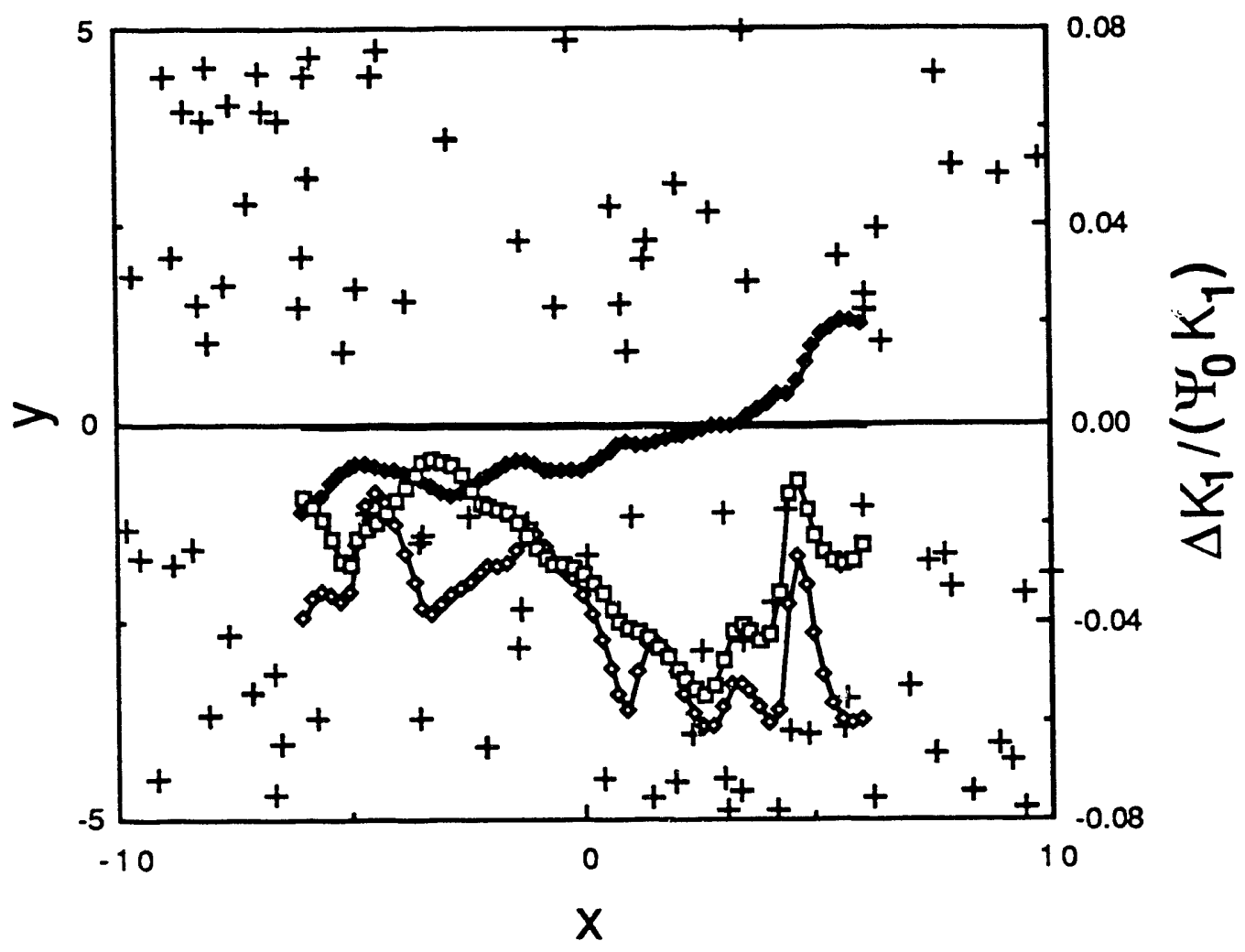

Figure 9. In this figure a crack was extended from left to right, starting at $x=-6$ to $x=+6$, in an array of whiskers located at + . The resulting change in mode $1 \mathrm{~K}$, expressed as $\Delta \mathrm{K}_{1} /\left(\psi_{0} \mathrm{~K}_{1}\right)$ are shown for the case where the whisker orientations in the array are uniformly random ( $\square)$, where the orientations are normally distributed around a mean of zero $(\diamond)$, and normally distributed around a mean of $\pi / 2(0)$. 


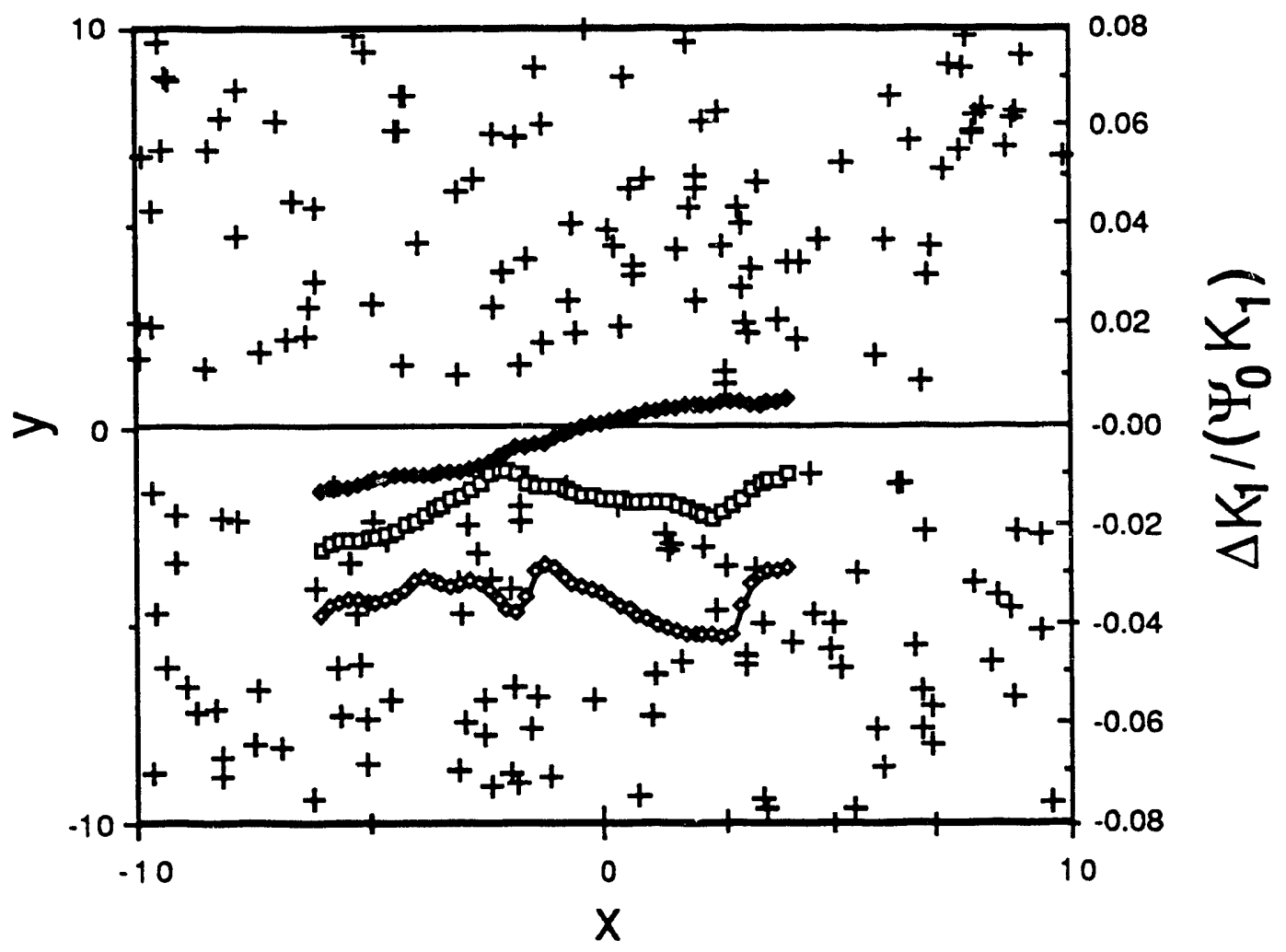

Figure 10. A situation similar to that in Figure 9 except: 1) the whisker locations are different, 2)the crack was extended from -6 to +4 , and 3 ) the model is twice as large, and therefore contains twice as many whiskers, as that in Figure 9. 


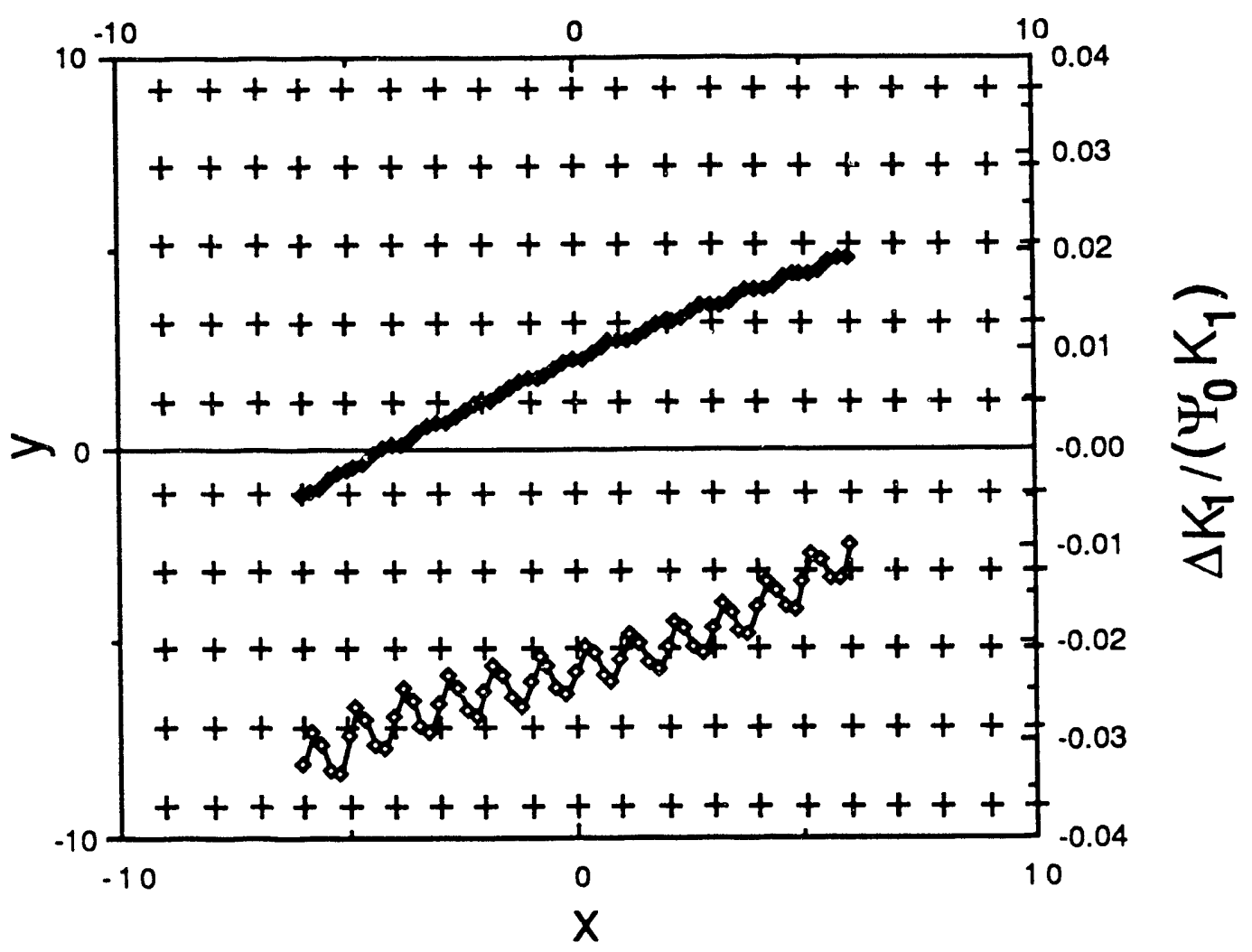

Figure 11. The results of extending a crack through a perfectly arranged rectangular array of whiskers. The whiskers are also perfectly oriented with the $(\bullet)$ results from whiskers parallel to the crack plane and $(0)$ perpendicular to the crack plane. 

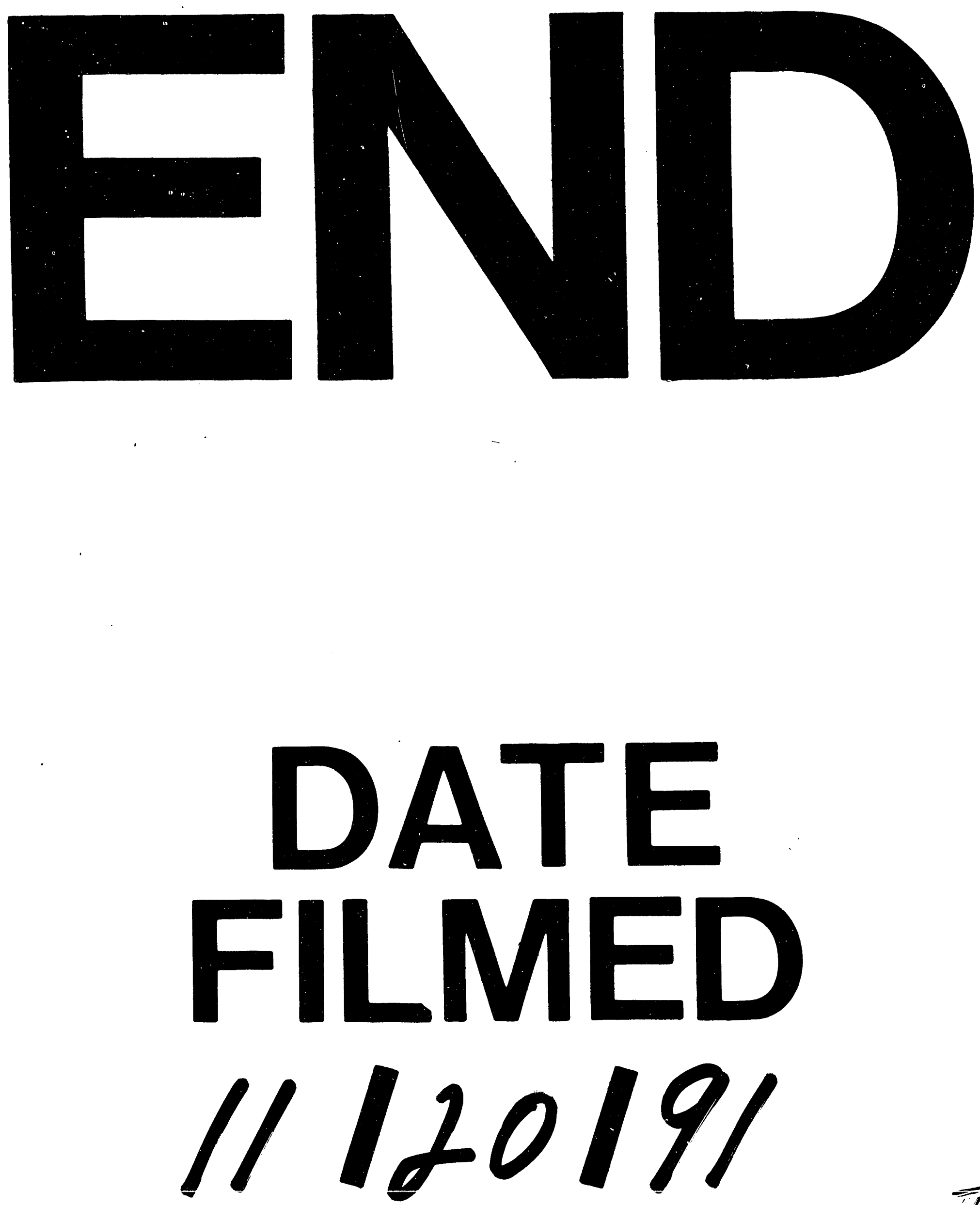
